Cahiers

Balkaniques

\section{Cahiers balkaniques}

36-37 | 2008

L'image de la période ottomane dans les littératures balkaniques

\title{
Barbare et infidèle
}

L'image de l'ennemi turc dans la guerre de 1876-1878 en Russie à travers la presse, les brochures de propagande, les correspondances et les mémoires

Barbarians and infidels: the image of the Turks in Russia during the war of 1876-1878

\section{Ilya Platov}

\section{OpenEdition \\ Journals}

Édition électronique

URL : https://journals.openedition.org/ceb/1571

DOI : 10.4000/ceb.1571

ISSN : 2261-4184

Éditeur

INALCO

Édition imprimée

Date de publication : 1 décembre 2008

Pagination : 293-320

ISBN : 978-2-85831-173-6

ISSN : 0290-7402

Référence électronique

\|lya Platov, «Barbare et infidèle », Cahiers balkaniques [En ligne], 36-37 | 2008, mis en ligne le 17 mai 2012, consulté le 06 juillet 2021. URL : http://journals.openedition.org/ceb/1571 ; DOI : https://doi.org/ 10.4000/ceb.1571

Ce document a été généré automatiquement le 6 juillet 2021.

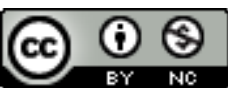

Cahiers balkaniques est mis à disposition selon les termes de la Licence Creative Commons Attribution - Pas d'Utilisation Commerciale 4.0 International. 


\section{Barbare et infidèle}

L'image de l'ennemi turc dans la guerre de 1876-1878 en Russie à travers la presse, les brochures de propagande, les correspondances et les mémoires

Barbarians and infidels: the image of the Turks in Russia during the war of 1876-1878

Ilya Platov

1 La présente étude a pour tâche de comprendre le phénomène de mobilisation patriotique des Russes en faveur de la libération des Slaves au cours de la crise balkanique et de la guerre russo-turque de 1877-1878, à travers l'étude de l'image de l'ennemi turc. La guerre nous apparaît en effet comme une période privilégiée, où se cristallisent les représentations de soi et de l'autre, et qui laisse des traces profondes dans la mémoire nationale ${ }^{1}$. La guerre de 1876-1878 a en effet été un facteur décisif dans la genèse du sentiment national après les grandes réformes de 1861, ainsi qu'un jalon important dans la constitution d'un imaginaire balkanique en Russie. En effet, en comparaison avec les conflits précédents, cette guerre présente une singularité inédite: la mobilisation politique des masses. La guerre est imaginée, anticipée et acceptée par la population qui s'impose ainsi en tant qu'acteur historique. Il est important de reconnaître que cette "nationalisation des masses » s'est réalisée sans aucun effort de propagande de la part de l'État impérial ${ }^{2}$.

2 Comment donc expliquer le fait que les contemporains aient été littéralement «empoignés» par la guerre, pour employer l'expression de l'historien Stéphane Audoin-Rouzeau $?^{3}$ En très grande partie parce que cette guerre est apparue à de nombreux contemporains comme une guerre juste, voire sainte, une vision fondée sur une certaine représentation de l'adversaire. L'« image de l'ennemi », est un élément constitutif d'un système de représentation qui forme un tout cohérent : le mythe de la croisade slave - la libération des Slaves orthodoxes des Balkans, devant aussi conduire à la régénération morale et spirituelle du peuple russe, est un alliage complexe et problématique, reflet d'une société placée entre la tradition et la modernité. De par ses principaux thèmes, il se situe, selon l'heureuse expression d'Alphonse Dupront, dans un 
" clair-obscur » idéologique : "Libération chrétienne, c'est le langage traditionnel, et pour tous valable; libération des nationalités, c'est le langage moderne, issu de l'universel révolutionnaire, libéral, langage de la diversité dans la fiction scrupuleusement maintenue d'une "européanisation commune" $»^{4}$. D'un côté, la croisade " contre » - le mythe s'appuyant sur une certaine image de l'ennemi et de sa victime innocente - et, de l'autre, la croisade " pour » : la liberté, la civilisation, la foi, et une quête de régénération nationale qui trouve une résonance au-delà du cercle des intellectuels panslaves. Nous allons étudier ici le mouvement qui conduit de la dénonciation des atrocités commises à l'encontre des Slaves du Sud vers un engagement qui est perçu comme un conflit de nature religieuse et justifié par des conceptions antagonistes du monde.

\section{L'impact des atrocités}

3 Ainsi que le remarque très justement Alphonse Dupront, « la société de croisade n'est à aucun moment la société des seuls croisés ensemble ; elle est la société des croisés et des infidèles ensemble [...] pour que la croisade soit, il faut qu'il y ait l'infidèle $»^{5}$. La représentation de l'ennemi transgressant les lois divines et humaines, qui focalise sur lui la haine et le rejet, est à la base du consensus patriotique dans les années 1876-1878. De fait, il est impossible de comprendre l'intensité de l'émotion dont la société russe est la proie à partir de la fin de l'année 1875 sans évoquer les récits d'atrocités, réelles ou imaginaires, commises par les Turcs à l'encontre des populations balkaniques d'abord, et contre les combattants russes ensuite. Ces récits font de la cruauté, caractéristique essentielle de l'ennemi, leur principal chef d'accusation. Pendant plus de deux ans, la population russe est soumise à une véritable surenchère d'images et de récits, qui ont la violence pour thème majeur. Il serait cependant réducteur de concevoir ceux-ci en termes de propagande, dans la mesure où ils sont le fruit d'une rencontre complexe entre la subjectivité collective et les faits transmis ${ }^{6}$. L'étalage des horreurs contribue à soulever des questions concernant la nature de la civilisation, de la justice et du bien. L'étude des récits d'atrocités est donc nécessaire pour comprendre les ressorts profonds de la mobilisation en 1876-1878.

\section{La « rhétorique de l'organe »}

4 L'impact de ces récits est indissociable de la mise en place de nouveaux moyens de diffusion de l'information, capables d'atteindre un large public. C'est de fait à travers la presse quotidienne que le public russe prend principalement connaissance de ces récits. L'émergence de cette presse marque la naissance d'une sphère publique où «l'opinion» peut s'exprimer, tandis que la presse quotidienne devient une arme puissante de création et de dissémination de nouvelles représentations de la nation ${ }^{7}$. Son impact est d'autant plus grand que la presse populaire, illustrée ou non, atteint pour la première fois un public très large. L'opinion russe s'est déjà intéressée à la guerre franco-prussienne, tandis que la répression de la révolte crétoise par les Turcs a soulevé son indignation en 1867. Le lancement de grands quotidiens tels que Novoe vremja est facilité par la demande de nouvelles en provenance des Balkans de la part de l'opinion russe ${ }^{8}$. La lecture de la presse progresse nettement dans les années 1870 et concerne un vaste public dans toutes les couches de la société 9 . La pratique des lectures collectives rend les nouvelles publiées accessibles même aux populations analphabètes, 
qui peuvent également regarder les images publiées dans les magazines illustrés. Les rumeurs qui circulent dans les milieux populaires reflètent souvent l'opinion de tel ou tel journal ${ }^{10}$. Les récits d'atrocités ont donc la capacité d'atteindre une audience très large, encore peu habituée à prendre ses distances vis-à-vis de la parole imprimée. La quantité même de ces récits produit un effet massif sur l'opinion et ce, dans les milieux sociaux les plus divers. Notre analyse se fonde sur un corpus très hétérogène, allant de la brochure de propagande aux correspondances et aux mémoires de témoins.

Comment aborder ces récits? John Horne a observé l'arrière-plan mythologique des récits "des mains coupées" qui circulent en France pendant la Première Guerre mondiale et qui, selon lui,

"[...] different d'un simple fait brut dans la mesure où elles réduisent une situation complexe et émotionnellement chargée à un personnage type, à une action type, qui symbolise la situation en la simplifiant ou la transformant. Ainsi, leur force réside dans sa puissance métaphorique, dans leur capacité d'évoquer par une image ou par une narration lapidaire, mais riche de signification, des émotions (généralement la peur) plus vastes et confuses $»^{11}$.

6 La charge émotionnelle de telles images tient à l'accumulation de stéréotypes culturels, d'images populaires et de références historiques qui leur sont préexistants, et qui leur confèrent leur puissance métaphorique. Ainsi, tous les récits de cruautés peuvent être, malgré leur profusion et leur diversité, ramenés à quelques thèmes majeurs, qui représentent les principaux axes du discours sur l'ennemi. Dans les pages qui suivent, nous allons analyser ce corpus de témoignages afin de dégager la présence, en leur sein, de ce que Laurent Gervereau a appelé une «rhétorique de l'organe»-la violence extrême en tant que lieu d'inscription d'une identité, car «les corps n'ont de toute façon pas la même valeur, suivant le camp, le sexe, l'âge, la partie atteinte, la blessure ou la mort $»^{12}$ - sur la période comprise entre 1876 et 1878 . Comme nous allons le constater, la minutie, l'abondance et la précision de détails caractérisent les descriptions de ceux qui veulent produire un effet de vérité, à une époque marquée par le positivisme ${ }^{13}$.

\section{Une barbarie sans limite}

7 Les récits des témoins, les articles des correspondants confortent l'image des Turcs sacrilèges, dont l'instrument favori est le pal, outil phallique et manifestation monstrueuse d'une sexualité bestiale et sans limite, souvent mise au compte de l'altérité religieuse : l'essence de l'islam, c'est la guerre à outrance, marquée par une violence qui affecte indistinctement femmes, hommes et enfants ${ }^{14}$. Dans un article publié à l'époque, le prêtre Apostol'skij compare les atrocités commises en Bulgarie aux montagnes de crânes dressées par Tamerlan. La décapitation est décrite comme un procédé particulièrement barbare. Depuis que V.V. Vereščagin a exposé les tableaux qu'il a peints en Asie centrale, la pratique est considérée comme une marque d'arriération, comme le signe distinctif d'une identité "orientale» et barbare. Aleksandr Benua reconnaît plus tard avoir éprouvé une certaine fascination pour les récits de tortures auxquelles ont été soumis les infortunés sujets de la Sublime Porte tombés entre les mains de «bachi-bouzouks sanguinaires $»^{15}$. Lesdits récits bénéficient d'une large diffusion et touchent même les enfants : A. Benua évoque également le cas d'une institutrice qui avait montré aux enfants de la crèche où elle travaillait des lubki représentant les « horreurs bulgares ». Les enfants auraient pleuré en les regardant. Un 
autre témoin de l'époque se souvient des expositions artistiques organisées régulièrement à Moscou avec, pour sujet principal, les atrocités commises au-delà du Danube ${ }^{16}$.

En 1876, la guerre serbo-turque sert de prétexte à l'exhibition de détails sordides censés caractériser la cruauté extrême des Turcs. En septembre 1876, Niva montre les supplices subis par ceux, Serbes ou Russes, que l'adversaire a faits prisonniers. L'image intitulée Prisonniers serbes, brûlés vifs par les Turcs représente trois hommes attachés à des arbres, avec un feu disposé sous leurs pieds. Leurs jambes sont entièrement carbonisées. Les visages des victimes expriment à la fois l'angoisse et la douleur extrêmes. Le réalisme de l'image vise à susciter l'indignation et la colère, ainsi que la compassion envers les victimes. Le commentaire de Niva qui accompagne cette image qualifie les faits "d'anachronisme monstrueux ", impensable à une époque qui se veut civilisée. Les Turcs ne respectent pas les conventions internationales, et ignorent les pratiques de la guerre entre nations civilisées. Les atrocités de ce genre sont le signe du caractère irrémédiablement corrompu des «adversaires héréditaires du christianisme $»^{17}$.

9 La véracité des faits de ce genre est d'autant mieux acceptée que les récits des témoins sont corroborés par les enquêtes minutieuses de journalistes indépendants et de représentants des gouvernements occidentaux. Le 23 juin 1876, le journal anglais Daily News commence à publier des descriptions détaillées des atrocités commises par les troupes irrégulières turques. Bien qu'elles trouvent une certaine résonance au sein de l'opinion publique anglaise, le gouvernement de Disraeli demeure sceptique. Le journal décide alors d'envoyer un émissaire pour mener une enquête sur le terrain. Le choix se porte sur l'américain A. MacGahan qui est déjà, à l'époque, un correspondant célèbre. Il mène une enquête particulièrement minutieuse en Bulgarie entre le 23 juin et le 20 novembre 1876, avec, pour résultat, une série de lettres dont la publication suscite plus de 300 manifestations en Grande-Bretagne ${ }^{18}$. L'ouvrage est très vite traduit en langue russe. L'introduction à l'édition russe souligne la crédibilité d'une enquête menée par un correspondant travaillant pour un journal privé et indépendant, qui a osé défier un grand empire au nom des principes d'humanité et de civilisation ${ }^{19}$. Ces lettres et leurs extraits sont publiés dans les journaux anglais et russes. A. MacGahan constate (en se fondant sur le rapport de Bering et de Skyler) que 79 localités habitées ont été détruites en Bulgarie et que le nombre total de victimes s'élève à 15000 . Il se présente comme un observateur objectif : loin de s'en tenir aux seuls récits des musulmans et des chrétiens, il a souhaité se rendre compte par lui-même de la véracité des rumeurs ${ }^{20}$. Les conclusions auxquelles il parvient rejoignent celles des journaux russes, toutes tendances confondues: les Turcs sont pires que des sauvages, car ils associent la décadence raffinée d'hommes éduqués et débauchés à une sensualité sauvage et sans limites $^{21}$.

\section{Violence et sexualité}

10 Le viol des femmes, manifestation d'une sensualité "orientale ", à la fois bestiale et sans limite, est un des thèmes les plus couramment exploités, fondé sur les témoignages publiés dans la presse, et couramment associé au thème du blasphème contre Dieu. Cette association vise de fait à montrer la manière dont l'ennemi transgresse aussi bien les lois divines que les lois humaines. On constate d'ailleurs une 
convergence de thèmes et de motifs entre les publications destinées à un public populaire et celles qui s'adressent à un public savant. A. MacGahan raconte l'histoire de Rajka, la « reine bulgare », battue et violée par les Turcs, alors qu'elle se préparait à une carrière d'institutrice. Fille de prêtre, et de surcroit institutrice, elle en vient à personnifier, à elle seule, le martyre de son peuple. Distinguée pour sa beauté et son intelligence, c'est après la mort de son père, assassiné dans son église, après avoir béni la «sainte cause " des insurgés ${ }^{22}$, qu'elle a pris part à l'insurrection. Dans ce cas, la barbarie de l'ennemi est rendue manifeste par son mépris de l'éducation. MacGahan précise d'ailleurs que les Turcs détruisent systématiquement les écoles. Dans un dessin de Karazin publié dans la revue Niva, intitulé Instant terrible. Une femme bulgare se cachant des bachi-bouzouks ${ }^{23}$, une jeune femme bulgare serrant un enfant entre ses bras se cache dans les buissons afin d'échapper à un cavalier turc. Le commentaire souligne qu'il s'agit d'une « scène banale » dans un pays livré à l'ennemi sanguinaire. Dans le but de renforcer l'effet de réalité, une précision indique en outre que ce dessin a été envoyé par un correspondant. Le cas est jugé représentatif de la nature sauvage de l'ennemi : "les Turcs sont assurément une grande nation, en ce qui concerne le pillage, le massacre et la bestialité ${ }^{24}$. Nombre de cas similaires montrent qu'une étroite correspondance a été établie entre l'atteinte au corps de la femme et la profanation du corps collectif de la nation. Sur les images, la Bulgarie est le plus souvent représentée avec les traits d'une femme. Dans une caricature publiée dans le journal satirique Budil'nik par exemple, la Bulgarie apparaît sous les traits d'une femme pendue à un arbre.

11 Le tableau de Konstantin Makovskij, réalisé en 1877, au titre suggestif de Bolgarskie mučenicy (Les martyrs bulgares) représente une scène qui devait être familière à ceux qui lisaient les journaux en Russie dans la mesure où ceux-ci assimilaient les outrages sexuels et le martyre religieux. Cette correspondance, que le titre du tableau ${ }^{25}$ tend déjà à établir, est encore renforcée ici par la localisation de la scène à l'intérieur d'une église saccagée, dont l'autel a été profané à la fois par le viol et le massacre. La première victime est une jeune femme bulgare, gisant nue et ensanglantée. Un évangile ouvert est posé sur son cadavre, la mort de la jeune femme étant assimilée au martyre pour la foi. Une autre jeune fille tenant un enfant dans ses bras est sur le point d'être dénudée par des bachi-bouzouks au faciès bestial. Le tableau n'évoque pas le viol directement, mais laisse plutôt au spectateur averti le soin d'imaginer la suite; par le mélange d'érotisme et de violence qui le compose, il est représentatif de cette production du temps de guerre ${ }^{26}$. Les agresseurs sont au nombre de trois; celui du milieu est de couleur noire, reflet probable de la rumeur qui court alors au sujet de la «légion égyptienne ", réputée plus féroce que les bachi-bouzouks eux-mêmes, l'éloignement géographique et l'exotisme qui lui sont associés étant en relation directe avec l'intensité de la violence. Viol, sacrilège, massacre des femmes et des enfants: le tableau se présente comme une véritable encyclopédie visuelle des récits publiés dans la presse, censée interpeller la conscience humanitaire et religieuse du spectateur. Exposé à l'Académie des Arts, il voit d'ailleurs défiler un grand nombre de visiteurs et alimente les conversations au sein de la société éduquée de Saint-Pétersbourg. D'après un témoignage, l'empereur lui-même aurait fondu en larmes devant le tableau au cours d'une visite de l'exposition ${ }^{27}$.

Mobilisant à la fois le thème national et religieux, l'exposition des horreurs lance un défi à l'honneur national russe, les racines communes des Russes et des Slaves étant en 
outre constamment rappelées. A. MacGahan lui-même ne s'est pas contenté de décrire les horreurs commises; il s'est aussi livré à une véritable enquête ethnographique en Bulgarie, au terme de laquelle il s'est rendu compte de la parenté linguistique et ethnographique entre les Bulgares et les Russes, peut-être dans l'intention d'influencer l'opinion russe: "Les peuples slaves se ressemblent beaucoup par l'expression du visage, la voix, les gestes. Quand le Russe, le Bulgare, le Serbe, le Monténégrin et le Tchèque parlent chacun leur langue maternelle, ils se comprennent sans peine $\aleph^{28}$. Afin de contrer les stéréotypes négatifs qui circulaient au sujet des Balkans en Occident, il se donne pour mission de prouver que les Bulgares appartiennent bel et bien à la civilisation européenne. Le journaliste se dit surpris par la propreté des maisons bulgares, "qui peuvent être comparées à un coin de France ou d'Angleterre». Les Bulgares ne sont pas des sauvages, affirme MacGahan, mais un peuple «industrieux, honnête, instruit et pacifique ${ }^{29}$; ainsi incarne-t-il les valeurs de la civilisation et de la bourgeoisie. L'identité des victimes, civilisées et semblables aux Russes, devient un enjeu national. Le publiciste et correspondant de guerre Evgenij Utin écrit que chaque jour qui apporte des faits nouveaux enfonce un peu plus profondément une lame dans le cœur de la Russie ${ }^{30}$.

\section{L'adversaire du christianisme}

13 Comme nous l'avons constaté, la violence s'exerçant contre la religion orthodoxe est un trait commun à tous ces récits. La violence dont fait preuve un adversaire fanatisé est toujours dirigée contre le peuple de Dieu, et elle vise à atteindre les victimes dans leur identité de chrétiens orthodoxes. Le prêtre N. Skrobotov publie en 1876 une brochure qui porte le titre Les martyrs chrétiens du $19^{e}$ siècle ou les brutalités turques en Bulgarie, où il évoque les exactions auxquelles sont soumises les populations bulgares, exactions qu'il n'hésite pas à comparer au martyre des chrétiens des premiers siècles ${ }^{31}$. Les histoires concernant le blasphème, la destruction des églises, ainsi que le meurtre et la torture des prêtres sont très fréquents. La crucifixion est une forme d'exécution souvent citée comme une preuve de la volonté de l'adversaire d'extirper la religion orthodoxe dans les Balkans ${ }^{32}$. Les actes de blasphème sont une accusation également très courante. Dans un de ses éditoriaux, M. Katkov cite le cas de musulmans ayant uriné dans les vases sacrés ${ }^{33}$. Les rapports de Skyler et de MacGahan décrivent des scènes d'immolation de femmes et d'enfants à l'intérieur des églises. Le journaliste déclare avoir vu des objets de cultes transformés en selles de cheval ${ }^{34}$, preuve supplémentaire que l'ennemi ne respecte ni l'innocence, ni Dieu. Il décrit une église profanée dans laquelle sont entassés des cadavres en décomposition, parmi lesquels on peut apercevoir des mains d'enfants. Le journaliste américain attribue cette férocité au fanatisme religieux : « le musulman qui tue un grand nombre d'infidèles est assuré de gagner sa place au paradis; Mahomet songeait sûrement aux guerriers, mais ses successeurs interprètent ses paroles d'une manière plus large $»^{35}$. La religion de l'ennemi est donc mise en cause.

Le meurtre brutal des consuls français et allemand par une foule de fanatiques dans une mosquée de Salonique est largement diffusé par la presse russe, et le journal Molva le qualifie d'acte de $"$ fanatisme sauvage ${ }^{36}$. Pour l'opinion russe, il s'agit d'une preuve supplémentaire de la nature barbare attribuée à l'islam. En effet, la colère de la foule a été provoquée par la protection que les deux consuls avaient accordée à une jeune fille bulgare qui allait être convertie de force à l'islam. Sankt-Peterburgskie vedomosti souligne 
que le fanatisme de la foule a été excité par le sermon d'un religieux local ${ }^{37}$. Une illustration dans Niva montrant la scène du meurtre met en scène une foule déchaînée, composée aussi bien d'hommes que de femmes, qui se livrent au massacre avec toutes sortes d'objets : lances, couteaux, barreaux arrachés aux fenêtres. Le commentaire précise que le consul français est mort après avoir reçu plus de trente-deux coups de poignard. La foule fanatisée s'est ensuite propagée dans la ville, en portant la sainte bannière de l'islam et en proférant des menaces à l'endroit de tous les chrétiens ${ }^{38}$. On voit ici encore à l'œuvre le processus d'identification d'un destin individuel à un destin collectif : la jeune fille slave refusant la conversion au péril de sa vie devient l'icône de la nation bulgare tout entière. La violence fanatique et irrationnelle est représentée comme étant directement suscitée par la religion de l'adversaire.

À travers ces récits, c'est la religion de l'ennemi qui est directement visée. Le correspondant russe N. N. Karazin parle des sauvages « qui ont retenu de leur Coran les sourates qui les incitent à piller, brûler et violer, ainsi qu'à détruire tout ce qui s'oppose à leur doctrine ${ }^{39}$. Un autre correspondant russe à Constantinople accuse les clercs musulmans d'inciter les foules au massacre: "quiconque aurait tué une dizaine de chrétiens ou bien un seul prêtre, gagne le paradis et le pardon des péchés ${ }^{40}$. V.V. Krestovskij rapporte la conversation qu'il a eue avec un imam, à la suite de la découverte d'un charnier à Telišs, en Bulgarie, en octobre 1877 :

"Après que l'officier chargé de l'interrogatoire, incapable de se retenir, lui ait ouvertement

reproché la sauvagerie des Turcs, l'imam lui avait répondu avec une conviction profonde:

Le Coran l'exige.

Mais vous devez respecter les normes du droit international, lui avait-il rétorqué.

Le Coran ne les reconnaît pas, répondit-il.

Mais vous avez bien signé la Convention de Genève?

Le Coran n'en dit pas un mot.

Ainsi, poursuit Krestovskij, ce fut là le dernier mot de l'imam, qui, avec son air aimable et

son caractère très posé, ne donnait même pas l'impression d'être un fanatique $»^{41}$.

V. I. Nemirovič-Dančenko écrit de Bulgarie que les Turcs sont mobilisés par une " guerre populaire » destinée à éradiquer les chrétiens des Balkans : « les musulmans y apporteront leur fanatisme et leur haine sans limites $»^{42}$. "Les prêcheurs itinérants venus de Constantinople excitent le fanatisme des habitants locaux $»^{43}$. Ces affirmations ont pour conséquence d'effacer toute différence entre les combattants et la population civile : les bachi-bouzouks ne sont-ils pas justement des civils armés? Le fanatisme est un principe d'explication du comportement des combattants adverses: un correspondant raconte comment, au cœur de la bataille de Plevna, un blessé turc qui avait été épargné par un soldat russe en avait profité pour l'attaquer dans le dos. Son acte est immédiatement mis au compte du fanatisme ${ }^{44}$. Le même V.I. NemirovičDančenko prétend avoir fait la rencontre d'un mollah « estimé à sa juste valeur et plus tard pendu par nous». Durant les massacres des Bulgares, ce religieux aurait massacré de ses propres mains plus de cent cinquante personnes à l'aide d'un bâton muni d'un crochet en cuivre. Le frère de l'artiste V. V. Vereščagin, Aleksandr, s'était ensuite emparé dudit bâton, en souvenir de la cause pour laquelle il avait combattu ${ }^{45}$. 


\section{Les récits de cruautés et la société russe pendant la guerre de 1877-1878}

Les actes de cruauté commis par les Turcs envers les blessés russes forment une autre accusation courante, et cet élément nous permet, à son tour, de mieux comprendre la détermination de la société russe à mener la guerre jusqu'à son terme malgré les revers subis. Alors qu'il ne constitue qu'un cas parmi d'autres, le cas des blessés russes horriblement mutilés et tués aux environs de Šipka, découverts ensuite par les Russes, mérite notre attention, en raison de la très large diffusion dont il a fait l'objet et des nombreux commentaires qu'il a suscités. L'image des corps mutilés connaît de fait une diffusion très large : elle est publiée à la fois dans Illustrirovannaja hronika vojny dans Niva, et dans des lubki des éditeurs Šarapov et Abramov. L'épisode figure aussi dans de nombreuses correspondances, comme une preuve du caractère inhumain de l'ennemi. Enfin, il est à l'origine d'un des plus célèbres tableaux de V. V. Vereščagin, Panihida po ubitym (Requiem).

Sur le lubok, la scène montre des généraux russes à cheval qui rendent un hommage solennel aux cadavres horriblement mutilés ${ }^{46}$. Le réalisme de ces images ne laisse aucun détail morbide de côté : des mains, des bras sont éparpillés à côté d'une montagne de têtes coupées. À gauche, un cadavre qui lève les bras, figés dans une expression de souffrance extrême, porte l'emblème de la Croix Rouge. À côté, des sacoches abandonnées, flanquées d'un croissant, permettent d'identifier les auteurs du massacre. La présence des correspondants français et anglais sur les lieux du massacre a, de fait, favorisé la célébrité de l'épisode, dont l'importance a pu être encore accrue par le fait que les massacres étaient imputés à l'armée régulière turque, pas seulement aux bachi-bouzouks. Effectuée dans le contexte des combats acharnés qui se tiennent alors autour de Plevna, cette publication a pour objectif de remonter le moral des troupes au front comme de ceux qui sont à l'arrière, en rappelant la malignité caractéristique de l'ennemi. Les commentaires des correspondants insistent aussi sur le fait que l'adversaire a visé les combattants en leur qualité de chrétiens. Le prince L. V. Šahovskoj, correspondant des Moskovskie vedomosti, indique par exemple que l'un des cadavres avait les doigts crispés en signe de croix ${ }^{47}$. Sur d'autres cadavres, les Turcs auraient taillé un crucifix alors que leurs victimes étaient encore vivantes ${ }^{48}$. V. I. Nemirovič-Dančenko évoque le récit d'un témoin qui lui a raconté comment les Turcs avaient composé un croissant avec les têtes des soldats suppliciés, tandis qu'une étoile, placée au centre, était composée par d'autres parties du corps ${ }^{49}$. Sur d'autres cadavres, les Turcs auraient coupé les organes génitaux, pour les enfoncer ensuite dans la bouche des morts. Telle qu'elle est représentée, la violence cherche à atteindre l'identité même des combattants: la virilité constitue une dimension essentielle du mythe de la croisade slave. Les soldats découverts par le correspondant étaient morts à la suite de tortures prolongées ${ }^{50}$. Les cadavres en viennent ainsi à représenter un lieu d'inscription de l'identité nationale.

Les descriptions n'épargnent aucun détail horrible. N. V. Maksimov décrit la manière dont une tête coupée, après avoir échappé aux mains du soldat chargé de l'enterrer, roule au bas d'une colline.

«La tête, réceptacle des plus nobles pensées de l'homme, était devenue semblable à un ballon, ramassée dans les champs comme de la pomme de terre! On n'était même pas capable de savoir à quels corps appartenaient ces têtes coupées, afin de rendre un dernier hommage aux camarades tombés! $»^{51}$. 
urcs sont aussi fréquemment accusés de violer les normes guerrières; selon une opinion très répandue (malgré la réserve de certains médecins), on leur reproche notamment d'utiliser des balles dum-dum ${ }^{52}$. V. I. Nemirovič-Dančenko, voyant les balles turques «s'enflammer » en touchant le sol, en conclut tout de suite qu'il s'agit de balles explosives $^{53}$. Des rapports de médecins militaires décrivent les blessures censées être dues à ce genre de balles. Le correspondant russe se plaint par ailleurs du fait que les rédactions des journaux russes ont, à son avis, tendance à épargner au lecteur les détails les plus horribles. Il cite le cas d'une " orgie de fanatisme ", au cours de laquelle les Turcs auraient brûlé vifs, dans une église, soixante-dix enfants du village de Čirpan. Les journaux avaient en effet passé le fait sous silence, "comme si ce genre de récits était destiné aux enfants ! ${ }^{54}$. Chacun de ces événements fait l'objet de rapports détaillés de la part des autorités militaires, rapports effectués, la plupart du temps, en présence d'observateurs étrangers.

21 L'image publiée dans Vsemirnaja illustracija suscite de nombreuses réactions dans des milieux très divers. Considérées dans leur ensemble, ces réactions témoignent du renforcement du consensus patriotique, fondé sur l'idée d'une guerre juste, menée contre un adversaire qualifié unanimement de barbare. V. V. Krestovskij évoque les réactions des soldats et des officiers russes présents lors de la scène. Un cosaque aurait exprimé l'opinion de tous, en promettant de ne plus épargner l'ennemi ${ }^{55}$. N. V. Maksimov, témoin du carnage, décrit ainsi ses sentiments: "Le désir de vengeance avait saisi tout mon être. D'un instant à l'autre, je suis devenu une bête féroce: j'avais envie de prendre un couteau et de dépecer le coupable, le dépecer lentement, alors qu'il était encore vivant, de me délecter de ses souffrances $\aleph^{56}$. En contrepoint de ce consensus patriotique, les grosses revues gardent une position réservée. La revue Otečestvennye zapiski réagit par un long article intitulé Lettres sur la guerre pour dénoncer la diabolisation de l'ennemi. De fait cette revue qui avait soutenu, dans un premier temps, le mouvement en faveur des Slaves, en vient à se ranger dans le camp des sceptiques dès avril 1877. L'auteur appelle à un jugement nuancé face aux «atrocités ", en distinguant les actes commis systématiquement par les formations irrégulières des actes occasionnels attribués à l'armée régulière et à la population musulmane. Il défend l'idée que les atrocités qu'ils commettent ne constituent pas une caractéristique essentielle des Turcs, découlant d'un fanatisme qui leur serait inné, mais que leur motivation doit plutôt être recherchée dans la volonté de défendre leur territoire à tout prix. Il met également en cause la presse qui attise la haine de l'ennemi par des «mystifications sensationnelles ${ }^{57}$. Pour l'auteur, la cruauté ne constitue pas l'apanage des peuples dits «barbares » : il mentionne la conduite des Anglais en Inde et les pogroms des juifs à Kiev, soupçonnés de vouloir échapper au service militaire. Convaincus que les Russes sont capables des pires atrocités, les Turcs croient aux fables véhiculées par la presse d'Istanbul, et c'est le désir de vengeance qui les anime qui les pousse à se conduire de manière brutale. L'opinion qui est ici exprimée représente cependant une opinion minoritaire : les grosses revues avaient vu leur audience chuter de manière spectaculaire durant la période du conflit ${ }^{58}$. Dans l'ensemble, ce sont les réactions d'indignation et de colère qui prédominent dans les témoignages de l'époque, qui confirment le stéréotype en place.

La large prolifération de ces récits, typifiés et stylisés, avant et pendant le conflit, alimente un discours de propagande. Les appels des Comités slaves, publiés dans divers 
organes de presse et également distribués auprès des églises, commencent généralement par l'évocation des atrocités commises (voir infra, chapitre II) ${ }^{59}$.

Ces textes modèlent une représentation de l'ennemi qui se propage aussi bien chez des combattants issus de la conscription que dans la société tout entière. Pour la majorité des témoins et des combattants, ces récits constituent l'unique source d'information au sujet de l'ennemi. L'étude des journaux, des mémoires et des correspondances atteste que ces récits ont été une source de craintes, qu'ils ont exacerbé la peur que pouvaient susciter des fantasmes liés à la mutilation ${ }^{60}$. Certains témoins considèrent le suicide comme un moindre mal, solution désespérée qui permet d'échapper aux mains de l'ennemi ${ }^{61}$. Il est difficile de mesurer l'impact de ces évocations fantasmatiques sur la conduite de la guerre du côté russe et sur la radicalisation de la violence guerrière qui en est la conséquence. Il semblerait que les combattants issus de milieux populaires aient été plus enclins à appliquer la loi du talion, comme l'indiquent certains correspondants. Selon un journaliste étranger, dont les propos sont cités par Otečestvennye zapiski, la guerre dans les Balkans devient de plus en plus sauvage : d'un côté, les Turcs font de la mutilation des blessés une pratique systématique : les 23 têtes coupées près de Šipka en sont la confirmation; d'un autre côté, le général Gurko est présenté comme étant également prompt à la besogne : il n'hésite pas à pendre une dizaine de Turcs, coupables d'avoir dissimulé des armes. Après les avoir sommés de rendre les armes, les cosaques poursuivent les Bachi-bouzouks sans merci; ni les uns ni les autres ne font de prisonniers ${ }^{62}$. Malgré la difficulté qu'il y a à trouver des faits précis, vu qu'ils sont généralement passés sous silence dans les mémoires, nous pouvons néanmoins constater que les récits que nous avons étudiés ont pour objectif, d'une part, de diffuser et d'ancrer une haine tenace à l'égard de l'adversaire et, d'autre part, d'éveiller et de renforcer la solidarité nationale et chrétienne. Les récits d'atrocités apparaissent donc comme le ciment le plus efficace et le plus durable de la mobilisation patriotique entre 1876 et 1878 .

\section{Le discours sur l'ennemi}

Le passage de la dénonciation des atrocités à la mise en place d'un discours sur l'ennemi peut être envisagé à l'aide de la notion de « seuil de l'intolérable ", mise en place par l'historien Alain Corbin. L'intériorisation de la césure de 1861 modifie l'attitude de l'obščestvennost' vis-à-vis de la violence jugée «tolérable », mutation de valeurs qui amorce, au cours du XIX ${ }^{\mathrm{e}}$ siècle, le déclin de l'expression publique de la violence extrême. Celle-ci est désormais condamnée en tant que pratique " asiatique » et en vient, soit à être reléguée dans un passé de barbarie, soit à être attribuée à un « autre » étrange et menaçant. La dénonciation des cruautés vise à mettre en cause le système politique, social, et religieux de l'Empire ottoman. Au cours de ce processus, la différence entre l'ennemi et soi tend à devenir une différence radicale ; l'ennemi est placé sous le signe d'une étrangeté monstrueuse nourrie de réminiscences littéraires, historiques et religieuses. Mais dans la mesure où cette différence implique aussi une distance prise à l'égard de son propre passé, elle conforte en même temps l'image d'une société qui aspire à être un membre à part entière du monde civilisé. Comme l'écrit Alain Corbin, « la perception d'une distance culturelle brutalement révélée par l'excès de cruauté permet à la société de calmer son angoisse par l'exécration des monstres ${ }^{63}$. La « rhétorique de l'organe » que nous avons analysée avait engendré, à la fois, un 
puissant sentiment de compassion envers les victimes et un sentiment d'indignation qui s'était bientôt transformé en impératif moral, exigeant une action volontariste.

\section{La figure de l'Oriental barbare} enracinés. Les récits d'atrocités nourrissent le discours sur l'ennemi, en lui fournissant l'argument central pour établir la différence irréductible qui sépare les « sauvages » des « civilisés ». Cet argumentaire s'appuie en réalité sur une longue tradition littéraire. Les représentations des Turcs, et plus particulièrement des bachi-bouzouks, s'inscrivent dans une longue tradition "orientaliste» russe dont Susan Layton a inventorié les principaux thèmes ${ }^{64}$. Comme en Occident, la littérature russe aime évoquer les merveilles de l'Orient indolent et sensuel, avec ses sérails et ses sultans, lieu d'une véritable contre-culture exotique dans l'imaginaire des Russes au XIX ${ }^{e}$ siècle ${ }^{65}$. L'image de l'Oriental «barbare ", qui transgresse les normes civilisées, est répandue au moins depuis l'époque de Puškin et de Bestužev-Marlinskij. Ainsi, la description du cadavre mutilé d'un soldat russe dans le Voyage à Erzeroum de Puškin présente des analogies frappantes avec certains des témoignages que nous avons cités précédemment, le recours à la mutilation corporelle étant montré comme la marque de l'esprit oriental ${ }^{66}$. La littérature joue un rôle déterminant dans la formation de la conscience nationale et, pour le lecteur éduqué en tout cas, de telles images ont, depuis longtemps, acquis la force d'un stéréotype, d'un jugement définitif. Dans la seconde moitié du XIX siècle, cet imaginaire se diffuse largement grâce à la presse, la littérature et l'imagerie populaire. La violence et la sensualité y sont toujours représentées comme deux attributs fondamentaux des tribus musulmanes, et cette image se précise au gré des conflits qui se prolongent dans le Caucase.

Ainsi, l'image du bachi-bouzouk peut être considérée comme une déclinaison du thème $\mathrm{du}$ « machisme oriental» (selon l'expression de S. Layton). Véritable personnification du mal, le bachi-bouzouk est de loin le personnage le plus représenté, tandis que les images figurant l'armée régulière ottomane sont beaucoup plus rares entre 1876 et 1878. Dans l'imaginaire des Russes, les bachi-bouzouks sont les volontaires du jihad, et constituent une image grotesque et inversée par rapport à l'image héroïque du volontaire russe ${ }^{67}$. Quand il s'agit de décrire leur apparence, c'est leur caractère inhumain qui est mis en avant, fondé sur l'écart par rapport aux idéaux classiques. George Mosse a montré en effet comment l'exaltation de la nation au XIX ${ }^{\mathrm{e}}$ siècle s'appuie sur une esthétique fondée sur les normes du classicisme ${ }^{68}$. La laideur des bachi-bouzouks se traduit par une absence de proportions des membres du corps : une tête démesurément grande, des yeux sauvages et fuyants. Il se distingue en outre par une pilosité anormale, et finit par apparaitre comme un sous-homme dont le visage exprime seulement l'ignorance et la sauvagerie ${ }^{69}$. Son habillement même, le costume oriental tarabiscoté et extravagant, est interprété comme une marque de l'altérité barbare $^{70}$.

Dans un numéro de Vsemirnaja illustracija, une gravure intitulée Bachi-bouzouks au combat offre une représentation typique de l'Oriental menaçant: le fusil entre les mains, la baïonnette entre les dents, le costume oriental, la posture menaçante, le regard cruel et fanatique traduisent une volonté de combattre jusqu'à la mort les ennemis de l'islam ${ }^{71}$. Une autre gravure montre aussi un bachi-bouzouk le couteau entre les dents, se livrant à des exactions à l'encontre des civils ${ }^{72}$. Les récits concernant

Cahiers balkaniques, 36-37 | 2008 
les bachi-bouzouks suscitent une curiosité qui est faite à la fois de répulsion et de fascination. Evgenij Utin évoque sa première rencontre avec des prisonniers turcs, « connus pour leurs méfaits à l'égard des civils bulgares ». L'un d'entre eux se vante de pouvoir déchirer des enfants en deux d'un seul coup ; il le décrit comme étant de taille immense, avec un visage d'une "beauté bestiale " ${ }^{73}$. La diffusion de ces stéréotypes provoque la curiosité des Russes au moment de l'arrivée des prisonniers turcs, comme en témoignent mémoires et journaux. Le journal Vsemirnaja illustracija place en couverture l'image de l'arrivée d'un convoi de prisonniers turcs dans une gare moscovite. D'après une circulaire du Ministère de l'intérieur, il y a plus de 9000 soldats turcs prisonniers en Russie entre 1877 et 1878, sans compter les officiers. Selon le témoignage de la presse, la population féminine, attirée par les récits évoquant leur sensualité bestiale est celle qui manifeste le plus de curiosité à leur endroit. En septembre 1877, Delo fait état du mouvement d'indignation qu'a provoqué, au sein de la société russe, le comportement de quelques dames prétendument philanthropes qui seraient allées jusqu'à savourer du champagne en compagnie d'officier turcs dans un parc de St Pétersbourg ${ }^{74}$.

Même le cadavre de l'ennemi n'est pas comme les autres. Son odeur est souvent mentionnée comme une manifestation symbolique de son altérité. Dans la brochure de propagande précédemment citée, on peut lire que les cadavres turcs dégagent une puanteur qui empeste une région entière du Monténégro ${ }^{75}$. L'idée qu'il existe une différence radicale entre l'odeur dégagée par un cadavre russe et un cadavre turc est une idée partagée aussi bien au front qu'à l'arrière. E. Ivanova, sœur de charité dans l'hôpital militaire de Fratešti, se souvient de l'odeur particulièrement abominable dégagée par les cadavres des ennemis décédés à la suite de leurs blessures ${ }^{76}$. $\mathrm{V}$. V. Vereščagin dans ses mémoires et V. I. Nemirovič-Dančenko dans sa correspondance s'attardent longuement à décrire les immondices d'un hôpital militaire turc découvert, aux alentours de Plevna ${ }^{77}$.

Parmi les dirigeants turcs, le sultan est fréquemment associé aux thèmes de la folie, qui est elle-même souvent attribuée à un excès de sensualité. Élevés dans les harems, au milieu d'intrigues, sans éducation, les sultans sont les auteurs de crimes abominables, des dépensiers sans limites, aux mœurs extrêmes; les descriptions de harems remplissent les pages des journaux ${ }^{78}$. Outre un exotisme évident, ces récits contribuent aussi à mettre en cause la légitimité même de l'Empire. Les coups d'État qui secouent la Sublime Porte en 1876 fournissent l'image parfaite d'un empire dominé par la violence, du sommet jusqu'à la base ${ }^{79}$. L'Empire ottoman est qualifié le plus souvent de "horde asiatique primitive ", qui ne peut même pas prétendre au titre de nation ${ }^{80}$. Les images de décadence qui lui sont associées illustrent le caractère "inorganique " de l'alliance des peuples qui le constituent. Il s'agit toujours de variations assez conventionnelles sur le sujet du « despotisme oriental », fréquemment discuté au XIX et au XXe siècle en Russie. La revue Delo consacre un long article à ce sujet. L'image de la Turquie y est présentée comme une antithèse de l'idée de nation, laquelle est, comme on le sait, imaginée comme étant à la fois fraternelle, homogène et souveraine ${ }^{81}$. L'article contient l'affirmation que « la Turquie n'a jamais été une nation: elle est un brassage disparate de plusieurs nations et de confessions religieuses avec un pouvoir central unique ». La Turquie est décrite comme une entité politique et sociale condamnée par l'histoire en raison de son système politique jugé archaïque ${ }^{82}$. Le statut du sultan, au-dessus des lois, en fait l'incarnation parfaite du despote oriental: commandeur des croyants, il est censé détenir le droit de vie et de mort sur ses sujets. Le peuple, privé de ses droits, 
existe pour son plaisir, et cette relation n'implique aucun devoir réciproque. Quel contraste avec la Russie, où l'empereur est au service de ses sujets ${ }^{83}$ !

\section{Un ennemi héréditaire?} la guerre contre les Turcs au sein des masses populaires. Le souvenir de la conquête mongole lui fournit une clef d'explication :

«La lutte contre l'infidèle, le Tatare, est un motif récurrent dans l'histoire du peuple russe [...] elle évoque les souffrances endurées, immortalisées dans les chants populaires et les récitations. Au cours des fêtes religieuses [...] Ce ne sont pas les faits historiques en tant que tels, mais l'ennemi lui-même, le mal lui-même qui paraissent familiers. Il n'y a pas d'ennemi plus populaire que le Turc $»^{87}$.

Ainsi, pour Aksakov, Tatare et Turc sont des termes interchangeables, tous deux étant déterminés par une appartenance commune à l'univers de l'infidèle, de l'islam. Effectivement, l'ethnonyme Tatare est, dans la culture populaire, un terme collectif qui regroupe l'ensemble des tribus asiatiques et non chrétiennes. Le terme est également synonyme d'ennemi tout $\operatorname{court}^{88}$. La conscience populaire qui se définit par la religion orthodoxe confond facilement Turcs et Tatares, offrant ainsi un terrain favorable à la propagande des Comités slaves. Les ennemis extérieurs sont désignés comme étant nehristi et busurmane, exclus des bienfaits de la loi chrétienne. La rumeur répandue dans la région de Kazan à la veille de la guerre russo-turque éclaire particulièrement bien notre propos: le bruit court en effet que les Tatares complotent un soulèvement en masse qui leur permettra de massacrer les Russes ${ }^{89}$. Comme l'atteste l'étude de la mémoire historique des paysans russes réalisée par A. V. Buganov, la conquête mongole était traditionnellement représentée comme un choc des mondes dont la religion orthodoxe constituait l'enjeu principal. Des paysans interrogés au milieu du XIX ${ }^{\mathrm{e}}$ siècle dans la région de Voronež et de Tambov avaient donné leur version de la conquête dans laquelle Batu conquérant aurait trouvé rassemblée face à lui la communauté orthodoxe tout entière ${ }^{90}$. La lutte contre un tel ennemi était représentée comme un enjeu pour l'existence même de la nation; la tradition lui prêtait en outre un caractère particulièrement brutal. Les chansons épiques russes, à la différence des chansons de 
geste d'Occident, se distinguent par l'absence totale de tout sentiment de respect pour l'ennemi, un constat qui s'applique d'abord et avant tout aux Tatares (Mongols). Comme l'affirme Vladimir Propp, les chansons russes ne possèdent pas d'équivalent du Sarrasin noble et généreux; la haine et le mépris envers les Tatares-Mongols constituent la seule attitude admise, dans la mesure où la lutte contre eux est une lutte pour la survie ${ }^{91}$.

La lutte contre les Tatares est aussi un sujet très courant des bylines et des chansons historiques ${ }^{92}$. Les différentes versions des bylines telles que Ilya Muromec et le tsar Kalin, comportent l'apparition des Tatares sous les murailles de Kiev et leur dispersion par les bogatyr ${ }^{93}$. Ce cycle des bylines était particulièrement apprécié du peuple. V. Propp a dénombré un certain nombre de sujets qu'il considère comme des invariants propres au cycle, tels que le surgissement brusque et dévastateur de l'ennemi, sa violence sauvage qui se manifeste par le meurtre commis sans discrimination, le blasphème qui consiste à détruire églises et monastères, ou encore, diverses offenses faites au sentiment religieux, tel l'ordre de faire enlever les croix des églises ${ }^{94}$. La violence sauvage de l'envahisseur mongol et la pratique qui consiste à capturer des esclaves sont fréquemment invoquées pour démontrer son caractère inhumain, et offrent des analogies frappantes et significatives avec la représentation des Turcs en 1876-1878. Pour ne citer qu'un exemple, on évoquera la coutume consistant à traiter l'ennemi de sobaka, c'est-à-dire de «chien ». Dans le cycle mentionné plus haut, c'est à Batu luimême que cette désignation est appliquée ${ }^{95}$. Par ailleurs, les noms des généraux turcs pendant et après la guerre russo-turque deviennent rapidement des termes péjoratifs : le nom Mukhtar, donné aux chiens, dérive du nom du général turc Mukhtar Pacha ${ }^{96}$. Comme l'indique B.A. Uspenskij, le chien, animal impur, est associé aux non-chrétiens à travers des expressions telles que sobaka tatarin («chien de Tatare»), ou bien des expressions comme pes'ja vera, "foi de chien », qui s'oppose à celle des «baptisés » et qui apparaît comme une entorse à la foi authentique ${ }^{97}$. Selon cette croyance, les Tatares n'auraient pas d'âme, puisque celle-ci est étroitement liée au concept de foi.

La tradition populaire connaissait par ailleurs des images telles que Le combat sanglant de Mamaj, sujet historico mythologique particulièrement apprécié dans les milieux populaires. Ainsi, La bataille de Kulikovo était en particulier un récit bien connu, ayant fait l'objet de maintes rééditions, dont une en 1869. Cette bataille était considérée par les paysans comme un épisode particulièrement important. L'image montre un épisode déterminant de l'histoire russe : la bénédiction de Dmitrij Donskoj par saint Serge, le duel entre le moine Peresvet et le Tatare Čelubej. Il est extrêmement significatif de voir que l'épisode de l'histoire russe retenu par la conscience populaire fait référence au combat contre un ennemi nomade et musulman. D. Rovinskij dit avoir vu des paysans pleurer en lisant le texte qui accompagnait l'image ${ }^{98}$. Il paraît dès lors naturel que la guerre turque ait été perçue de manière similaire. De fait, des analogies formelles existent entre cette série d'images et celles qui représentent les bachi-bouzouks dans les illustrations destinées au public populaire. Les cavaliers bachi-bouzouks ressemblent de manière frappante à l'image conventionnelle du Mongol, cavalier nomade se livrant à la destruction et au massacre, dans la mémoire populaire. Sur le dessin publié dans Mirskoj vestnik, intitulé « La rage des bachi-bouzouks dans un village serbe ", sont représentés trois cavaliers qui ont des traits mongols ${ }^{99}$. On voit bien comment, à travers ces exemples, des parallèles s'opèrent, porteurs de suggestions et de mises en relation susceptibles de nous éclairer quant au succès de la mobilisation 
patriotique dans les couches sociales les plus larges. La confrontation de ces récits et de ces images montre que celle-ci s'appuie sur des référents culturels qui puisent dans une mythologie nationale construite à travers une lecture particulière du passé historique.

L'image de l'ennemi nous permet de comprendre comment la société éduquée russe, qui veut oublier le servage, projette sur l'adversaire son anxiété de manquer elle-même aux standards civilisés. Les Russes ne sont-ils pas eux-mêmes des barbares asiatiques aux yeux des Européens ? Par un mouvement qui trahit un complexe d'infériorité face à l'Occident, les milieux éduqués découvrent la vocation de la Russie à être le portedrapeau de la civilisation européenne en Asie, une idée soutenue par Dostoïevski dans un article postérieur à la crise ${ }^{100}$. L'évocation des massacres donne lieu à un véritable renversement de valeurs qui porte la Russie au sommet du monde civilisé. Selon le jugement d'un contemporain, "c'est le barbare russe qui défend l'humanité et le christianisme, tandis que le chrétien anglais est du côté de la barbarie, de la tyrannie et $\mathrm{du}$ mal $»^{101}$. Pour les Russes, le problème touche de près la question de l'identité nationale. La rencontre et le conflit avec l'Asie étaient inscrits dans la trame même de leur histoire, à travers les mythes que nous avons évoqués plus haut. À cette occasion, la société russe pouvait se soustraire aussi bien à la tutelle occidentale qu'aux servitudes du passé. Le Turc devient l'incarnation de l'ordre ancien, auquel une société émancipée cherche à se soustraire. Que l'ennemi soit vu à travers la figure quasi mythique du Tatare ou celle, plus récente, du despote oriental, dans tous les cas, il est envisagé comme un obstacle à la libération ultime, l'obstacle au passage vers une société une et régénérée par son retour à l'ordre originel. Exprimée dans un langage accessible au plus grand nombre, l'efficacité du stéréotype consiste à établir une communauté de consentement, un remède à la crise que traversait la société russe de l'époque.

36 Le philosophe russe Vladimir Solov'ev, dans son dernier ouvrage intitulé Trois entretiens sur la guerre, la morale et la religion, publié en 1898, met en évidence le mécanisme que nous avons décrit ci-dessus en exposant un épisode particulièrement atroce de la période de $1877-1878^{102}$. Dans un plaidoyer en faveur de la guerre juste, le général, un des principaux personnages, évoque la vengeance à laquelle il s'est livré après avoir été confronté au spectacle de corps d'Arméniens sauvagement mutilés. L'extrait est tellement représentatif qu'il mérite d'être cité :

«Je ne voyais le monde qu'avec répugnance et j'agissais comme machinalement [...] Alors il se fit tout à coup une lumière en moi [...] Mon cour avait fondu, pour ainsi dire, et c'était comme si le monde me souriait de nouveau... De nouveau, j'agissais machinalement ; mais dans mon âme il y avait une sorte de légèreté: c'était comme si j'avais des ailes. Je savais ce qu'il fallait faire, avec une assurance absolue, et je sentais que ce serait fait sans faute [...] et le Seigneur bénit mes six décharges, sans exception. J'avais dans mon âme comme une fête de Pâque lumineuse... et un silence, une légèreté inconcevable, comme si on m'avait lavé de toutes les impuretés de l'existence et débarrassé de toutes les pesanteurs terrestres; enfin, un état proprement paradisiaque; je sentais la présence de Dieu, et de Lui seul $»^{103}$.

Cet extrait semble donner raison à Alain Besançon, qui écrit au sujet des grandes guerres qui jalonnent l'histoire russe : «La menace extérieure résout le conflit, apaise l'angoisse, rend le sujet à sa vérité et à sa liberté ${ }^{104}$.

On constate à l'issue de cette analyse que l'image de l'ennemi forgée au sein de la guerre de 1876-1878 contribue à renforcer le consensus patriotique dans une société qui subit l'impact des médias de masse. Les représentations de l'ennemi qui structurent cette «culture de guerre » sont un mélange étonnant d'idées traditionnelles et 
modernes. La multiplication des acteurs sociaux qui participent à la mise en forme de la représentation de l'ennemi et la diversification des vecteurs de diffusion font que l'opinion participe de plus en plus à la définition des objectifs et des idéaux de guerre.

L'effervescence de l'imaginaire national ne s'est pas traduite par une plus grande cohésion de la société. En 1876, le pouvoir est critiqué pour sa passivité face aux massacres; la diffusion d'images de cruauté extrême et d'un stéréotype de l'ennemi indique une certaine «brutalisation » de la société russe ${ }^{105}$. Ce climat explique en partie la flambée de la violence révolutionnaire qui submerge le pays entre 1878 et 1881 . La notion selon laquelle il serait juste de se battre contre un adversaire cruel et oppresseur peut facilement se transformer en appel à la violence révolutionnaire. En effet, dès 1877, certains organes émigrés appellent à combattre les «bachi-bouzouks de Saint-Pétersbourg $»^{106}$.

La représentation de l'ennemi, de la victime slave souffrante et du territoire balkanique, forgée au cours de cette guerre fait désormais partie de la mémoire collective des Russes. Les mêmes images et les mêmes récits resurgissent en 1912 (au cours des guerres balkaniques), ainsi que pendant la Première Guerre mondiale. À partir de 1914, les lubki et les illustrations montrent des scènes d'atrocités turques très semblables à celles que nous avons étudiées. Les thèmes « orientalistes » sont exploités par le cinéma naissant qui reprend le thème de la supériorité culturelle, morale et religieuse des Russes sur un monde oriental décadent ${ }^{107}$. L'écho de cette guerre résonne jusqu'à aujourd'hui, comme en témoignent les réactions de l'opinion russe face à la guerre au Kosovo. Le souvenir de la croisade slave semble s'animer à chaque fois qu'atteinte est portée aux « frères de foi et de sang » Slaves.

\section{L'Armée du Danube. L'arrière-garde des armées turques qui battent en retraite}

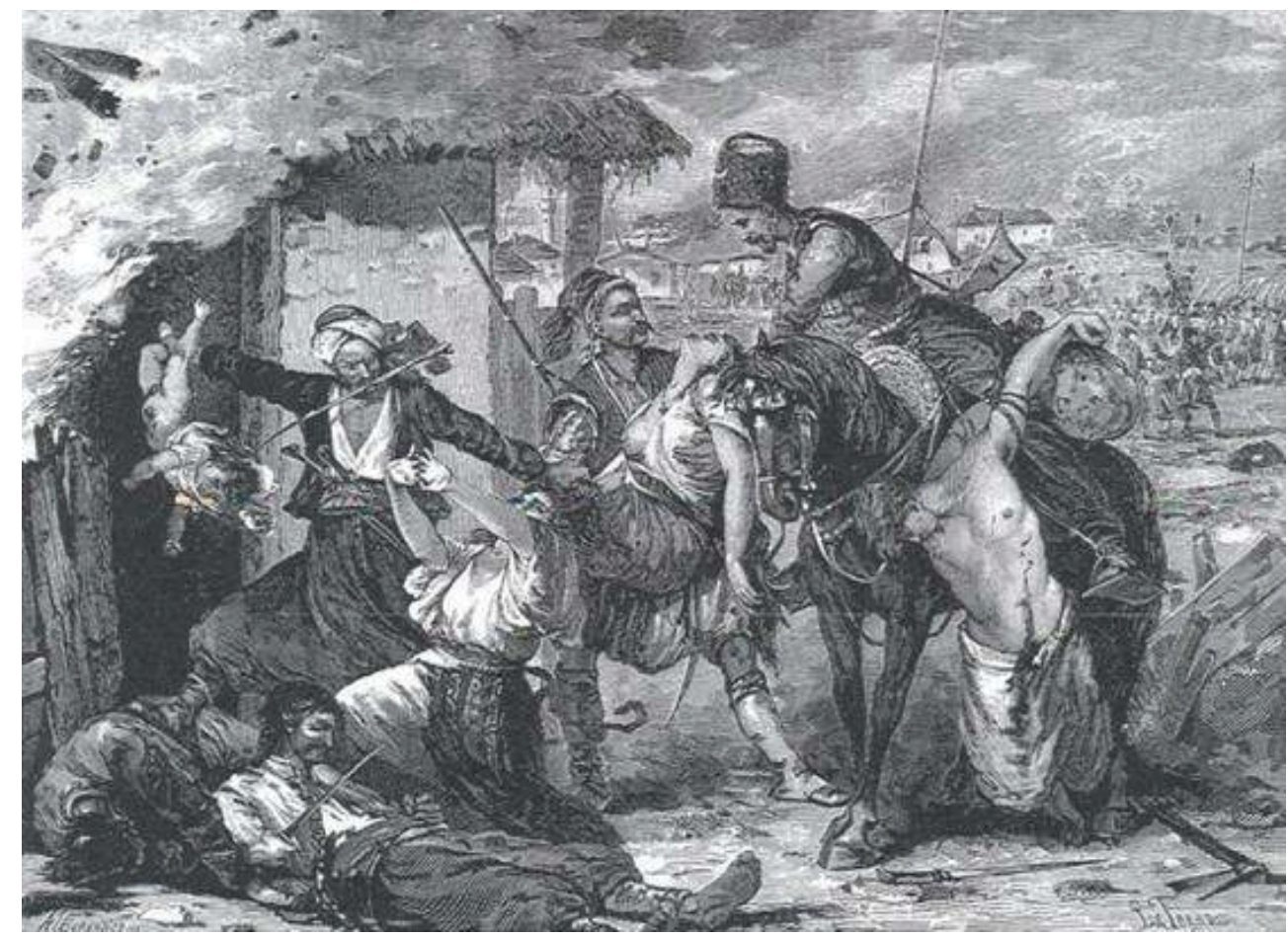

(artiste : F. Gaanen, Illustrirovannaja hronika vojny, $n^{\circ} 61,1877$ ) 
Les Bachibouzouks au combat

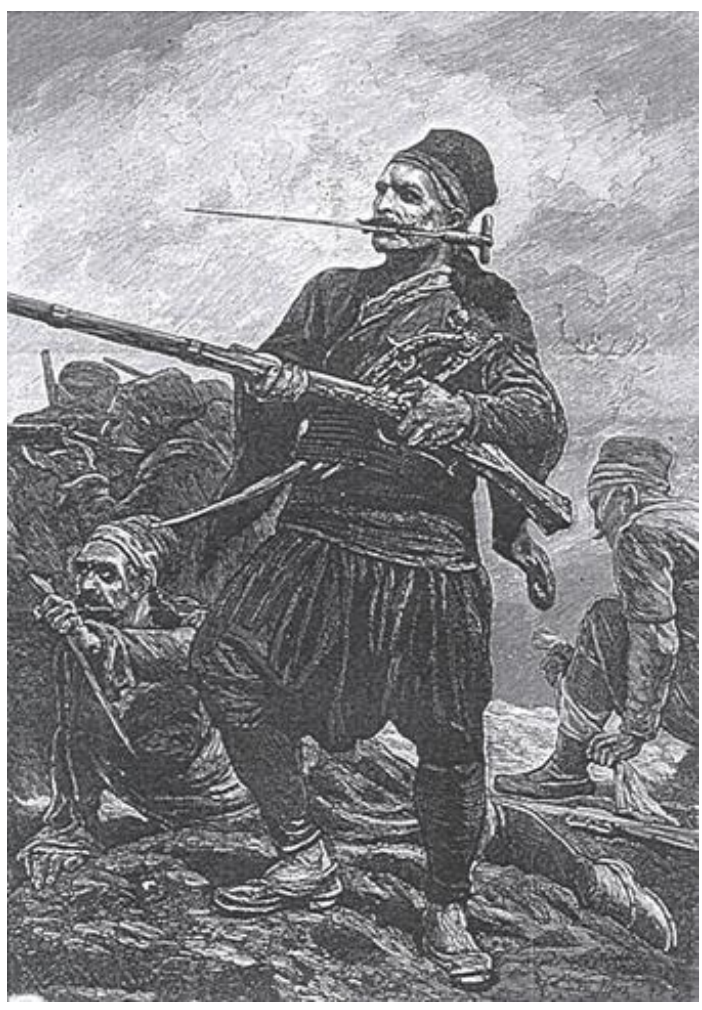

(artiste : A Daugel', Ibid., n 47, 1877)

\section{Les ennemis du christianisme}

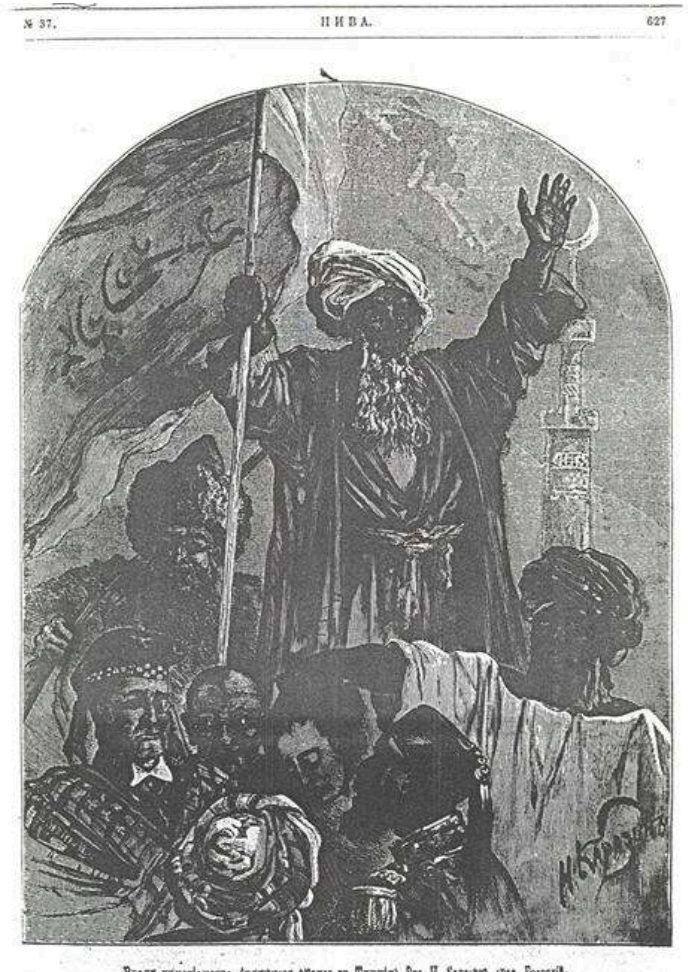

(artiste : N. N. Karazin, Niva, n 37, 1876) 
L'armée du Danube. Le détachement du général Gurko dans les Balkans découvre les corps mutilés des combattants russes
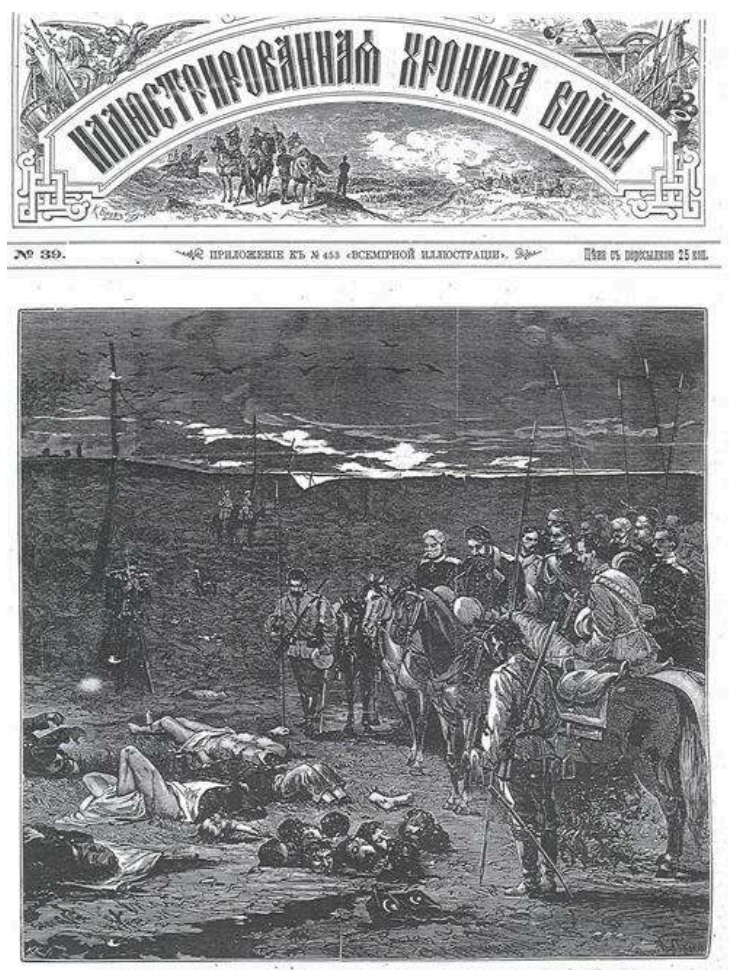

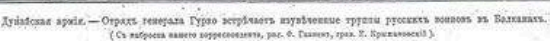

(artiste : F. Gaanen, Illustrirovannaja hronika vojny, nº 39, 1877).

\section{NOTES}

1. A. D. Smith, "War and ethnicity: the role of warfare in the formation, self-images and cohesion of ethnic communities", Ethnic and Racial Studies, octobre 1981, volume 4, nº 4, p. 375-397.

2. Le terme de "nationalisation des masses » est employé pour la première fois par G. L. Mosse pour désigner l'entrée des masses sur la scène politique par l'intermédiaire de mythes et de symboles à travers leur participation aux rituels collectifs. Cf. G. L. Mosse, The Nationalization of the Masses: Political Symbolism and Mass Movements in Germany from the Napoleonic Wars through the Third Reich, Ithaca \& London: Cornell University Press, 1991.

3. S. Audoin-Rouzeau, A. Becker, 14-18, Retrouver la guerre. Paris : Gallimard, 2000, p. 113 sq.

4. A. Dupront, Le mythe de croisade, Paris : Gallimard, 1997, t. 1, p. 533 ; t. 2, p. 933.

5. A. Dupront, Le mythe..., op. cit., t. 3, p. 1430.

6. J. Horne, «Les mains coupées : "atrocités allemandes" et opinion française en 1914 », in Guerre et cultures, 1914-1918, Paris : Armand Colin, 1994, p. 134.

7. S. McReynolds, The News Under Russia's Old Regime: the Development of a Mass-Circulation Press, Princeton: Princeton University Press, 1991, p. 3.

8. Ibid. pp. 79-82. 
9. Cf. J. Brooks, When Russia Learned to Read: Literacy and Popular Literature, 1861-1917, Princeton: Princeton University Press, 1985.

10. Aleksandr Èngel'gardt écrivait que les rumeurs populaires reflétaient non seulement les faits, mais aussi les opinions publiées dans les journaux. A. N. Engel'gardt, Iz derevni. 12 pisem 1872-1887, M., 1987, pp. 299-300.

11. J. Horne, « Les mains... », op. cit., p. 139.

12. L. Gervereau, Les images qui mentent. Histoire du visuel au $X X^{e}$ siècle, Paris : Seuil, 2000, p. 92.

13. T. W. Laqueur, "Bodies, Details, and the Humanitarian narrative", in The New Cultural History, Berkeley: University of California Press, 1989, pp. 176-204.

14. P. Apostol'skij, "Slavjanstvo i pravoslavie», Pravoslavnoe obozrenie, novembre 1876, $\mathrm{n}^{\circ} 11$, pp. 528-539.

15. A. Benua, Moi vospominanija, M., 1980, t.1-2, p.365. Les bachi-bouzouks, formations irrégulières utilisées par les autorités ottomanes, forment une milice armée qui échappe au contrôle des militaires turcs.

16. D. Nikiforov, Moskva v carstvovanie Aleksandra II, M., 1904, p. 23.

17. Niva, 1876, $\mathrm{n}^{\circ} 38$, p. 643, 645.

18. A. D. MacGahan, Zverstva v Bolgarii. Pis'ma special'nogo korrespondenta « Daily News ", Spb., 1877, p. ii-iii. Parallèlement, une enquête officielle est menée, en juillet 1876, par l'anglais Bering et le consul américain Skyler, qui parviennent à des conclusions similaires. Le même Skyler est l'auteur d'un ouvrage en deux volumes consacré aux atrocités commises par les Russes au Turkestan. La revue Otečestvennye zapiski, dans le compte-rendu de cet ouvrage, mentionne que les faits relatés «faisaient pâlir ceux commis par les Tcherkesses et les bachi-bouzouks" : Otečestvennye zapiski, octobre 1876, $\mathrm{n}^{\circ}$ 10, p. 206.

19. A. D. MacGahan, Zverstva..., Ibid., p. iii. Dans son enquête, le journaliste américain s'est fondé sur les témoignages des consuls étrangers présents sur les lieux, des missionnaires américains, des employés des chemins de fer ainsi que des victimes ayant survécu aux massacres, ainsi que des Turcs eux-mêmes.

20. Ibid. p. 3.

21. Ibid.p. 137.

22. Ibid. p. 37.

23. Niva, 1876, n³9, p. 707.

24. Ibid.

25. Pour un équivalent poétique, voir le poème Bolgarka (La Bulgare) de Ja P. Polonskij.

26. V. M. Garšin avait critiqué l'érotisme selon lui déplacé et situé à la limite de la banalité : «La Bulgare martyrisée par les bachi-bouzouks [...] est "savoureuse", la peinture révèle certainement la main d'un grand maître. Mais comment avait-il donc usé de son talent ? Le sujet dont s'était emparé Mr. Makovskij constitue l'un des drames les plus atroces qui puisse arriver [...] une mère, dont l'esprit est obscurci par l'effroi, voit son enfant se vider de son sang; l'artiste a néanmoins trouvé bon d'y insérer des "accessoires" destinés à faire saliver les concupiscents jeunes et vieux », V. M. Garšin, Sočinenija, M.-L., 1960, p. 339.

27. A. Benua, Moi..., op. cit., p. 365.

28. Ibid. p. 16.

29. Ibid. p. 24.

30. E. Utin, Pis'ma iz Bolgarii v 1877 g., Spb., 1879, p. 2.

31. Cf. N. A. Skrobotov, Hristianskie mučeniki XIX stoletija ili neistovstva turok v Bolgarii, Spb., 1876.

32. Voir, par exemple, F. M. Dostoïevski dans le Journal d'un écrivain de septembre 1876. Pour d'autres récits au sujet de prêtres crucifiés, voir par exemple Novoe vremja, 25 juillet $1876, \mathrm{n}^{\circ} 145$, p. 2, ainsi que Russkij Mir, 26 juin 1876, $\mathrm{n}^{\circ}$ 174, p. 1.

33. Moskovskie vedomosti, 16 septembre 1876, $\mathrm{n}^{\circ} 235, \mathrm{p} .1$.

34. E. Utin, Pis'ma..., op. cit., p. 54.

Cahiers balkaniques, 36-37 | 2008 
35. Ibid. p. 26.

36. Molva, 2 mai 1876, $\mathrm{n}^{\circ} 18$, p. 1.

37. Sankt-Peterburgskie vedomosti, 6 mai 1876, $\mathrm{n}^{\circ} 124, \mathrm{p} .1$.

38. Niva, 1876, $\mathrm{n}^{\circ} 21$, p. 372.

39. N. N. Karazin, Dunaj v ogne. Dnevnik korrespondenta. V 2-h častjah, 1905, p. 255. Le même auteur avait publié dans Niva en 1876 une gravure intitulée Les ennemis du christianisme (scènes de rue en Turquie). Celle-ci est dominée, en son centre, par l'imposante figure d'un religieux turc qui prêche la guerre sainte devant une mosquée, visible à l'arrière-plan. La main gauche levée, la bannière de la guerre sainte dans la main droite, il personnifie le fanatisme religieux. À sa gauche, se trouve un bachi-bouzouk qui porte le fusil à l'épaule, tandis qu'à droite, un autre brandit la tête coupée d'un chrétien. Le dessin est censé représenter le massacre barbare comme étant la conséquence du jihad islamique.

40. Moskovskie vedomosti, 6 juillet 1876, $\mathrm{n}^{\circ}$ 170, p. 2.

41. V. V. Krestovskij, Dvadcat' mesjacev v dejstvujuščej armii, 1877-1878. Pis'ma v redakciju gazety « Pravitel'stvennyj vestnik », t. 2, Spb., 1879, p. 238.

42. V. I. Nemirovič-Dančenko, God vojny (Dnevnik russkago korrespondenta, 1877-1878), t. 1, Spb., 1879, p. 30.

43. Ibid. p. 31.

44. Ibid. p. 25.

45. Ibid. p. 70.

46. Le seul écart entre l'illustration publiée dans Niva et La chronique illustrée de la guerre d'un côté, et les lubki de l'autre concerne la présence parmi ces derniers de Skobelev, devenu déjà un véritable héros populaire.

47. L. V. Šahovskoj, Dva pohoda za Balkany, M., 1878, pp. 23-24. L'auteur mentionne à nouveau le même fait à un autre endroit, p. 130.

48. Ibid. p. 129.

49. V. I. Nemirovič-Dančenko, God..., t. 1, op. cit., p. 12.

50. Ibid. p. 25.

51. N. V. Maksimov, Dve vojny 1876-1878 gg. Vospominanija i rasskazy iz sobytij poslednih vojn, Spb., 1879 , t. 2, p. 476.

52. V. I. Nemirovič-Dančenko, God..., t. 2, op. cit., p. 59 ; 87-88.

53. Ibid.p. 320.

54. Ibid., pp. 185-186.

55. V. V. Krestovskij, Dvadcat'..., t. 1, op. cit., p. 439.

56. N. V. Maksimov, Dve..., t. 2, op. cit., p. 475.

57. «Pis'ma o vojne », Otečestvennye zapiski, septembre 1877, $\mathrm{n}^{\circ}$ 9, p. 123.

58. S. McReynolds, News..., op. cit., p. 82. D'après certaines sources, le refus publiquement affiché d'acheter les grosses revues et les journaux sceptiques tels que Golos avait été considéré par certains comme un geste patriotique.

59. « Ot S.-Peterburgskago slavjanskogo blagotvoritel'nogo komiteta », Delo, août 1876, pp. 4-6.

60. L V. Šahovskoj, Dva..., op. cit., p. 57.

61. V. I. Nemirovič-Dančenko, God..., t. 2, op. cit., p. 47.

62. «Pis'ma o vojne », Otečestvennye zapiski, septembre 1877, $\mathrm{n}^{\circ}$ 9, p. 130.

63. Cf. A. Corbin, « Du Languedoc aux cultures sensibles », in Pour une histoire...., op. cit.

64. Cf. S. Layton, Russian Literature and Empire: Conquest of the Caucasus from Pushkin to Tolstoy. Cambridge: Cambridge University Press, 1994.

65. O. Figes, Natasha's..., op. cit., p. 384.

66. Je remercie Mme le professeur Laure Troubetskoy pour avoir attiré mon attention sur cet épisode. 
67. Faisant pendant à l'atmosphère qui régnait en Russie, le gouvernement turc était accusé d'attiser une atmosphère de croisade. Un auteur anonyme décrivait ainsi une de ces processions qui étaient une image inversée des manifestations qui se déroulaient alors en Russie : « Une foule de volontaires turcs en haillons, un derviche en tête, défilait lentement dans la rue. Brandissait une bannière verte, le derviche hurlait qu'il n'est dieu que Dieu et Mahomet est son Prophète ", cité dans Bor'ba za nezavisimost' stražduščih slavjan Balkanskogo poluostrova, M., 1877, p. 157.

68. Cf. G. L. Mosse, The Nationalization..., op. cit.

69. Bor'ba..., op. cit., p. 159.

70. Ibid.p. 159.

71. Ibid. p. 376.

72. Illustrirovannaja hronika vojny, $1878, \mathrm{n}^{\circ} 61, \mathrm{p} 88$.

73. E. Utin, Pis'ma..., op. cit., pp. 45-46.

74. Delo, septembre 1877, $\mathrm{n}^{\circ}$ 9, p. 120.

75. Ibid. p. 238.

76. E. Ivanova, « Poezdka vo Fratešti. Iz putevyh vpečatlenij ‘sestry’ », Slovo, mai 1878, p. 164.

77. V. V. Vereščagin, Na vojne. Vospominanija o russko-tureckoj vojne 1877 g. hudožnika V. V. Vereščagina, M., 1902, pp. 172-173 ; V. I. Nemirovič-Dančenko, God..., t. 2, op. cit., pp. 52-60.

78. Dans une des brochures parues à l'époque, il est écrit que le Sultan Abdul Medjid « abusait du harem, et malgré le fait qu'il se nommait commandeur des croyants, buvait sans retenue », cité dans Bor'ba..., op. cit., p. 205.

79. Comme l'écrit le journal Syn otečestva en 1876, «les scènes sanglantes de l'insurrection se combinent avec des drames non moins sanglants à Constantinople même », cité dans Syn otečestva, 9 mai $1876, \mathrm{n}^{\circ} 19$, p. 1 .

80. M. M. Vozdviženskij, « Slavjanskij vopros », Pravoslavnoe obozrenie, janvier 1877, nº 1, p. 189.

81. B. Anderson, L'imaginaire national. Réflexions sur l'origine et l'essor du nationalisme, Paris: La Découverte, 1996, pp. 20-21.

82. Syn otečestva, Ibid. p. 450.

83. A. M., « Čto spasaet Turciju ?», Delo, septembre 1876, n 9, p. 238.

84. Cf. O. Figes, Natasha's Dance. A Cultural History of Russia, New York: Metropolitan Books, 2002, pp. 364-365.

85. Ibid.p. 376.

86. On pourrait aussi méditer sur le rôle providentiel de " fléau de Dieu », que Danilevskij avait conféré, dans son « historiosophie », aux hordes nomades en général. Les Huns, les Mongols et les Turcs sont semblables à des comètes, dont le passage apporte la destruction et qui disparaissent ensuite sans laisser de traces. Ils ont cependant un rôle instrumental dans l'économie de la Providence, favorisant la prise de conscience d'une nation, n'étant eux-mêmes que des phénomènes éphémères.

87. I. S. Aksakov, « Reč' vice-prezidenta Moskovskogo slavjanskogo blagotvoritel'nogo komiteta v zasedanii 24 oktjabrja 1876 goda », Pravoslavnoe obozrenie, décembre 1876, n 12 , pp. 833-834.

88. V. Ja. Propp, Russkij geroičeskij èpos, M. : «Labirint», 1999, p. 407. L'expression siluška zla nevernaja, qui signifie littéralement la «force du mal infidèle », est, elle aussi, communément employée. L'auteur précise aussi que «terre russe » et «foi chrétienne » sont, dans les bylines, quasi synonymes.

89. A. V. Buganov, M. M. Gromyko, O vozzrenijah russkogo naroda, M. : « Palomnik », 2000, p. 507.

90. Ibid.p. 480.

91. Vladimir Ja. Propp, Russkij..., op. cit., p. 398.

92. Ibid. p. 10.

93. Ibid.p. 305.

94. Ibid., pp. 312-317. 
95. Ibid. p. 355. Dans la tradition russe, le chien est un animal impur, dont l'aboiement est comparable au juron et au blasphème, «impropres aux chrétiens orthodoxes " selon un mandement ecclésiastique vieux-russe. Pour plus de détail, voir B. A. Uspenskij, "L'aspect mythologique des jurons russes - II ", in I. Lotman, B. A. Uspenskij, La sémiotique de la culture russe, Paris : l'Age d'Homme, 1990, pp. 425-461.

96. A. V. Buganov, M. M. Gromyko, O vozzrenijah..., op. cit., p. 510. Ce nom est d'ailleurs toujours en usage, et l'auteur de ce travail peut témoigner qu'il est attribué de préférence à de grands chiens particulièrement féroces, mais obéissant à leurs maîtres. Ne peut-on pas induire, à titre d'hypothèse, que s'exprime ainsi une forme de domination symbolique de l'infidèle par le chrétien?

97. B. A. Uspenskij, « L'aspect.... », op. cit., pp. 436-427.

98. Cité dans A. F. Nekrylova, Russkij batal'nyj lubok, «Art», 1999, nº 3, p. 98.

99. Mirskoj vestnik, 1876, $\mathrm{n}^{\circ}$ 9, p. 272.

100. O. Figes, Natasha's..., op. cit., p. 380.

101. V. I. Nemirovič-Dančenko, God..., t. 1, op. cit., p. 210.

102. Dans sa préface, l'auteur explique que son récit est fondé sur un fait réel dont il a été informé par le général M. M. Bibikov qui avait participé à la campagne de 1877-1878.

103. V. Soloviev, Trois entretiens sur la guerre, la morale et la religion, Paris : O.E.I.L., 1984, pp. 71-73.

104. A. Besançon, Le tsarévitch immolé. La symbolique de la loi dans la culture russe, Paris : Librairie Plon, 1967, pp. 142-143.

105. Pour le concept de «brutalisation ", voir G. L. Mosse, Fallen Soldiers. Reshaping the Memory of the World Wars, $2^{\text {e }}$ ed., New York: Oxford University Press, 1991, pp. 159-182.

106. Cf. Z. K. Ralli, Bašibuzuki Peterburga, Genève, 1877.

107. H. F. Jahn, Patriotic Culture in Russia during World War I, Ithaca and London: Cornell University Press, 1998, p. 19.

\section{RÉSUMÉS}

La compréhension de la mobilisation patriotique des Russes en faveur de la libération des Slaves du Sud au cours de la crise balkanique et de la guerre russo-turque de 1876-1878 nécessite la prise en compte des récits de cruautés relayés par la presse et par d'autres vecteurs d'information qui aboutissent à la cristallisation d'une représentation de l'ennemi à la fois barbare et infidèle. Pendant plus de deux ans, la population russe est soumise à une véritable surenchère d'images et de récits qui ont la violence pour thème majeur. La dénonciation des atrocités débouche sur la justification d'un conflit de nature essentiellement religieux et justifié par des conceptions antagonistes du monde. La construction de l'image de l'ennemi associe des thèmes traditionnels et modernes et s'appuie sur un système de représentations déjà solidement enracinés.

The author argues that the patriotic mobilization of the Russians in favor of their Slavic brethrens during the Balkan crisis and the Russian-Turkish war of 1876-1878 was to a large extent based on various stories of atrocities committed by the Turks against the Slavs propagated by the press and other medias. For over two years, the Russians were exposed to an avalanche of images and stories with gruesome violence as their main theme. The Turks were represented as "barbarians" and "uncivilized" on the one hand, and as "enemies of Christianity" on the other. 
The denunciation of atrocities led to the justification of an essentially religious conflict based on mutually exclusive world-views. The image of the enemy thus created associates both traditional and modern themes, and is based on the bedrock of shared representations.

\section{INDEX}

Index géographique : Russie

Mots-clés : presse russe, Bachi-bouzouk, Makovskij Konstantin (1839-1915), Bogatyr, propagande nationaliste, Byline, nationalisme, Makovski Konstantin (1839-1915), violence, opinion publique

Keywords : russian press, propaganda, Russia, Politics, History, Literature, Makovskij Konstantin (1839-1915), violence, public opinion

motsclesmk ОТОМАНСКАТА ИМПЕРИЈА

motsclestr Rusya, Osmanlı İmparatorluğu

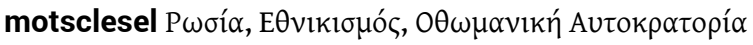

Index chronologique : dix-neuvième siècle, Empire ottoman

Thèmes : Sciences politiques, Histoire, Littérature 